\title{
De la Regio au Regnum. L'improbable « nation des Aquitains » au premier Moyen Âge
}

\section{Adrien Bayard}

\section{OpenEdition}

\section{Journals}

Édition électronique

URL : http://journals.openedition.org/ifha/8032

DOI : $10.4000 /$ ifha. 8032

ISSN : 2198-8943

\section{Éditeur}

IFRA - Institut franco-allemand (sciences historiques et sociales)

\section{Édition imprimée}

Date de publication : 31 décembre 2014

ISSN : 2190-0078

\section{Référence électronique}

Adrien Bayard, «De la Regio au Regnum. L'improbable « nation des Aquitains » au premier Moyen Âge », Revue de l'IFHA [En ligne], 6 | 2014, mis en ligne le 31 décembre 2014, consulté le 20 avril 2019.

URL : http://journals.openedition.org/ifha/8032 ; DOI : 10.4000/ifha.8032

Ce document a été généré automatiquement le 20 avril 2019.

(CIFHA 


\title{
De la Regio au Regnum. L’improbable "nation des Aquitains " au premier Moyen Âge
}

\author{
Adrien Bayard
}

1 Héritière du système provincial romain, l'identité de l'Aquitaine au premier Moyen Âge se serait affirmée en réaction à la formation du royaume wisigoth et arien de Toulouse, puis confortée dans une opposition frondeuse au pouvoir franc. Pour contrôler cet espace, les souverains carolingiens n'auraient eu d'autre choix que de créer un royaume d'Aquitaine dans les années 780-781. Tel est depuis la fin du XIXe siècle, le cadre d'analyse de l'historiographie française pour cet ensemble géographique couvrant un large quart sudouest de la Gaule, qualifié pour le début du VIIIe siècle de «seul état romain d'Occident » et dont les éléments saillants seraient la force de l'héritage antique et le caractère particulièrement remuant de l'aristocratie ${ }^{1}$. Or les travaux entrepris ces vingt dernières années démontrent que loin de constituer des marqueurs d'identité, ces traits révèlent surtout des fonctionnements communs aux sociétés altimédiévales². Il semblait donc pertinent d'interroger les processus de construction de cette périphérie diversement intégrée au monde franc, en envisageant successivement: les contextes d'utilisation des termes Aquitania et Aquitani chez les auteurs au très haut Moyen Âge ; la structuration du discours sur l'identité de cette région au VIIIe siècle; et enfin la création d'un espace politique par la jeune royauté carolingienne.

\section{L'Aquitaine au très haut Moyen Âge, quelle réalité ?}

2 Pour les VIe et VIIe siècles, on est frappé par le très faible nombre des occurrences d' Aquitania et Aquitani dans la totalité des corpus, aussi une attention particulière doit-elle être portée à chacune de ces mentions si l'on veut pouvoir cerner la réalité décrite par les auteurs derrière ces termes. Grégoire de Tours, lui-même originaire de cet espace, n'utilise qu'une seule fois le mot d'Aquitania ${ }^{3}$, pour décrire dans ses Dix livres d'Histoire les 
violences exercées par Euric, roi des Goths, à l'encontre des chrétiens de Novempopulanie et des deux Aquitaines. Or, cette unique mention est en elle-même problématique, car il s'agit d'une correction des éditeurs, le texte parlant des deux Germanies. Cet usage du substantif Aquitania fait clairement référence aux deux provinces romaines centrées sur Bourges et Bordeaux, d'autant qu'elles sont ici associées à la Novempopulanie. L'emploi de ce nom dans un contexte de géographie administrative s'inscrit dans la continuité des auteurs du Ve siècle, plus particulièrement la correspondance de Sidoine Apollinaire ${ }^{4}$. Pour l'évêque de Tours, le cadre spatial de référence reste la petite partie, ou civitas. On trouve, en effet, dans ses œuvres une soixante de références à la cité tourangelle, plus de vingt occurrences de la civitas arvernensis et une dizaine de mentions de la ville de Poitiers. Un passage illustre la force de cette appartenance civique : Grégoire y rapporte les paroles du prêtre Riculf, qui, sûr de s'être débarrassé de son évêque, se vante d'avoir enfin libéré la ville de Tours des Auvergnats ${ }^{5}$. Le poète et futur évêque de Poitiers Venance Fortunat, lorsqu'il décrit son périple en Gaule, rappelle que les limites des deux Aquitaines sont la Loire et la Garonne ${ }^{6}$. Cet extrait semble témoigner de la prégnance des structures territoriales du Bas-Empire à la fin du VIe siècle, moins comme des réalités politiques que comme des structures d'appréhension de l'espace.

La première mention des Aquitani comme une gens, une " nation ", date du VIIe siècle. Elle se trouve dans la Chronique de Frédégaire, où les Aquitains figurent entre les Gaulois et les Bretons $^{7}$ dans la longue liste des gentes issues des fils de Noé et de la confusion des langues. Ce texte est essentiel, car il est le premier à présenter l'Aquitaine comme une entité politique, un royaume confié à Charibert, frère cadet de Dagobert en $629^{8}$. Il convient cependant de ramener cet ensemble à sa juste mesure. Car si le chroniqueur décrit un espace allant de la « Loire aux limites de l'Espagne et du pays des Wascones aux Pyrénées $»^{9}$, il insiste dans un deuxième temps sur le fait que l'autorité de Charibert se limitait en réalité à son siège toulousain et aux pagi de Cahors, d'Agen, de Périgueux et de Saintes, le contrôle des zones entre la Garonne et les Pyrénées étant purement théorique (fig. 1). Cette création associant pour la première fois Toulouse à l'Aquitaine, semblait répondre à deux impératifs pour Dagobert: confier un royaume à son frère, pour sauvegarder l'apparence d'un partage de l'héritage de Clotaire II ; mais surtout créer sur la Garonne un grand commandement susceptible d'intervenir rapidement en Septimanie wisigothique et de rappeler aux Wascones leur soumission aux rois des Francs. L'action des successeurs de Charibert, le patrice Félix, puis le duc Loup, s'inscrit dans cette ligne de conduite. Ainsi, ce dernier est lié au maire du palais Ébroïn et ne réunit un concile à Bordeaux (670-673) qu'avec l'autorisation du roi Childéric II $^{10}$. Sous la plume de Julien de Tolède, c'est comme relais du pouvoir mérovingien dans le sud de la Gaule que Loup agit pour soutenir les ducs Hildéric et Paul en Septimanie contre le roi Wamba ${ }^{11}$. Si la première moitié du VIIe siècle voit l'apparition dans les sources écrites d'une gens des Aquitains, il faut replacer cette évolution dans le contexte du développement des discours identitaires en Gaule, parallèlement à la création des duchés périphériques par les souverains mérovingiens ${ }^{12}$. 


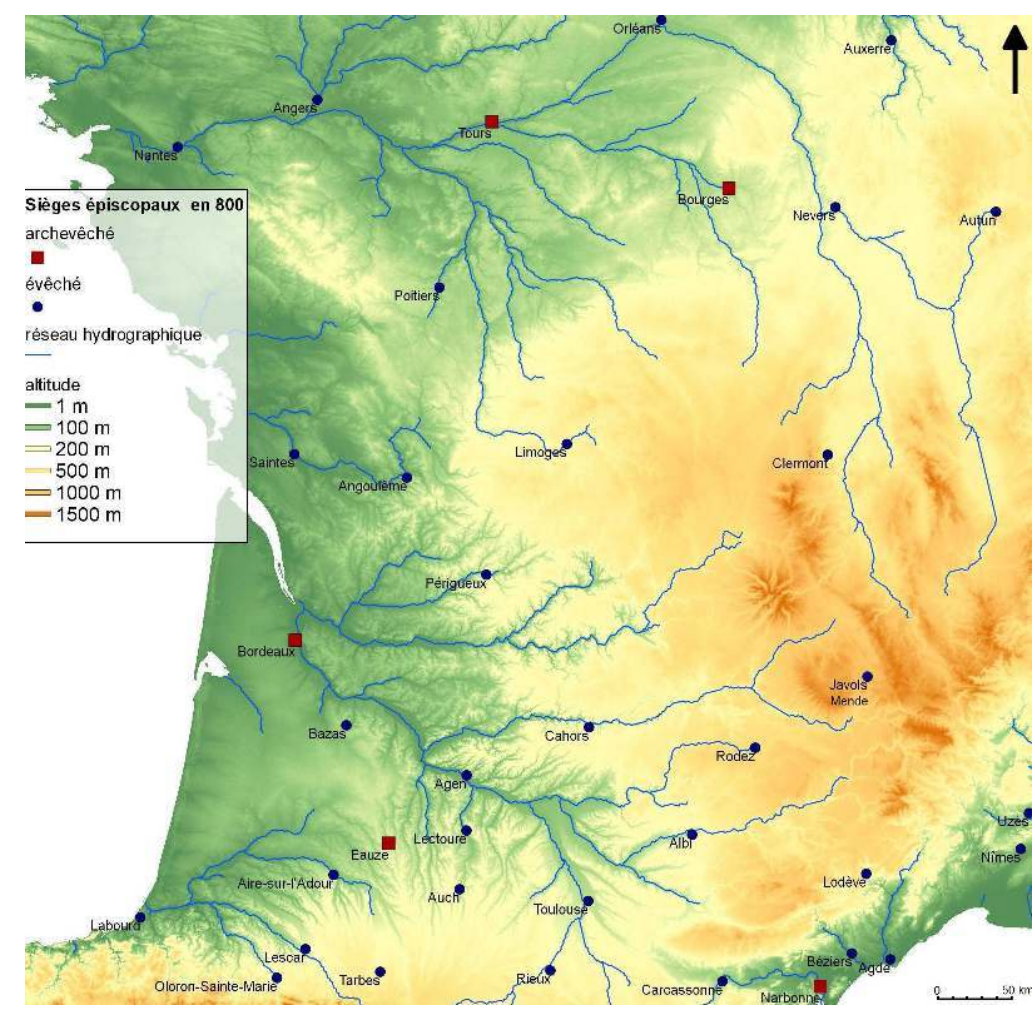

Figure - Les sièges épiscopaux dans le sud-ouest de la Gaule (C) Adrien Bayard

C'est dans ce cadre que l'identité « romaine » des espaces entre Loire et Garonne doit être discutée. Car, si les élites locales utilisent le terme de romanos pour se décrire, il s'agit véritablement d'une identité sociale. Cette dernière est fondée sur le mythe d'une continuité parfaite des familles sénatoriales, largement adossée à la fonction épiscopale. On trouve les traces les plus tangibles de ces constructions identitaires dans la littérature hagiographique des VIIe et VIIIe siècles, où s'épanouit la figure du saint aquitain de haute extraction $^{13}$. Ainsi, dans la Passio Praejecti, l'auteur justifie l'élection contestée de Praejectus sur le siège de Clermont en 662, en rappelant que l'impétrant est issu d'« une noble ascendance romaine" déjà distinguée par l'ancienneté de son adhésion au catholicisme et par les miracles dont elle a pu bénéficier ${ }^{14}$. Or, le reste du document permet de reconstituer autour de cet évêque des réseaux familiaux, dépassant largement la cité de Clermont et l'Aquitaine. Cependant l'hagiographe a choisi de mettre en avant ces éléments discursifs, qui donnaient au prélat le vernis social attendu d'un candidat à l'épiscopat de ces régions. Ce substrat culturel fut conforté par les maires du palais et les premiers souverains carolingiens ${ }^{15}$ qui utilisèrent pour cela le droit romain. La rédaction des formulaires dans l'espace ligérien au VIIIe siècle ${ }^{16}$ pourrait correspondre à cette volonté de fixer une personnalité juridique. Mais il est important de noter que la production de ce discours identitaire fut très largement exogène.

\section{Production d'un discours identitaire externe}

Les années 730-760 voient le développement des références à l'Aquitaine et aux Aquitains, mais ces dernières se trouvent systématiquement dans un contexte conflictuel. Cette période est en effet marquée par une intense compétition au sein des élites du monde franc, plus particulièrement entre les Pippinides et les groupes contrôlant les duchés 
périphériques. Or, nos sources (Continuations de la Chronique de Frédégaire et Annales regni Francorum) proviennent toutes des cercles fidèles aux maires du palais d'Austrasie. Il n'est donc pas étonnant de voir progressivement s'installer une confusion entre les Aquitains, « les Romains au sud de la Loire » et les Wascones ${ }^{17}$. Cette fusion entre deux gentes jusquelà bien distinctes s'effectue par étapes à partir de 740. Elle sert à masquer l'opposition d'une faction de l'aristocratie au pouvoir de Charles Martel et de ses successeurs, en assimilant cette partie des élites du monde franc à une gens rebelle à l'autorité royale depuis le VIIe siècle.

6 Le caractère polémique de notre documentation a également pour effet de créer une unité par défaut des populations d'Aquitaine, qui se seraient dans leur ensemble opposées aux Francs, en se rangeant derrière les ducs "nationaux». Or, une étude serrée des sources disponibles permet de très largement déconstruire ce modèle. Dans un premier temps, il convient de rappeler que les ducs présents en Aquitaine sont issus de l'administration mérovingienne et souvent liés au maire du palais de Neustrie ${ }^{18}$. Dans les années 720, le plus puissant d'entre eux, Eudes, est désigné dans le Liber Pontificalis comme «dux Francorum, princeps in Aquitania $»^{19}$. De même, les Pippinides n'ont pas systématiquement une attitude hostile à leur égard. Pour les deux successions connues, ce sont les maires du palais puis les rois qui choisissent, au sein d'un groupe familial en train de se structurer, celui qui va exercer la charge ducale (figure 2). Ainsi, en 735 Charles Martel se rend au castrum de Blaye et choisit Hunoald, plutôt que son frère Hatto, pour lui remettre le ducatus en échange d'un serment de fidélité ou promissio fidei $i^{20}$. Charles, puis Pépin, ont donc cherché des interlocuteurs stables dans une optique de " coopétition " ${ }^{21}$, alternant collaboration et rivalité avec les mêmes partenaires, dans la tradition des relations entre les souverains francs et les princes périphériques. Ces derniers semblent d'ailleurs très loin de contrôler la totalité des groupes aristocratiques en Aquitaine. Il n'existe que deux éléments témoignant d'une volonté de ces ducs de sortir du cadre territorial défini en 629 : il s'agit d'un extrait des Miracles de saint Martial ${ }^{22}$ (VIIIe-IXe siècles), relatif à la prise de contrôle de Loup à Limoges; ainsi qu'une charte brivadoise de $757^{23}$, concernant la rétrocession d'une villa située en Limagne et concédée en précaire à un fidèle de Hunoald. Ces éléments permettent de mesurer la distorsion pouvant exister entre le contexte de rédaction et les événements décrits. Il faut également tenir compte du fait que les textes se copient mutuellement, reprenant les mêmes trames narratives. Aussi, si les années 730-780 voient la naissance d'un discours des chroniqueurs royaux sur la gens des Aquitani, la fin du VIIIe et le IXe siècle consacrent sa normalisation. L'exemple le plus frappant de ce phénomène peut être observé dans l'utilisation du qualificatif par excellence des gentes opposées aux Francs, perfidiae. Pour les annalistes, seuls les ducs sont initialement jugés perfides ${ }^{24}$, puis progressivement les comtes qui les entourent sont également désignés comme perfidi, enfin ce trait devint caractéristique des Aquitains ${ }^{25}$. 


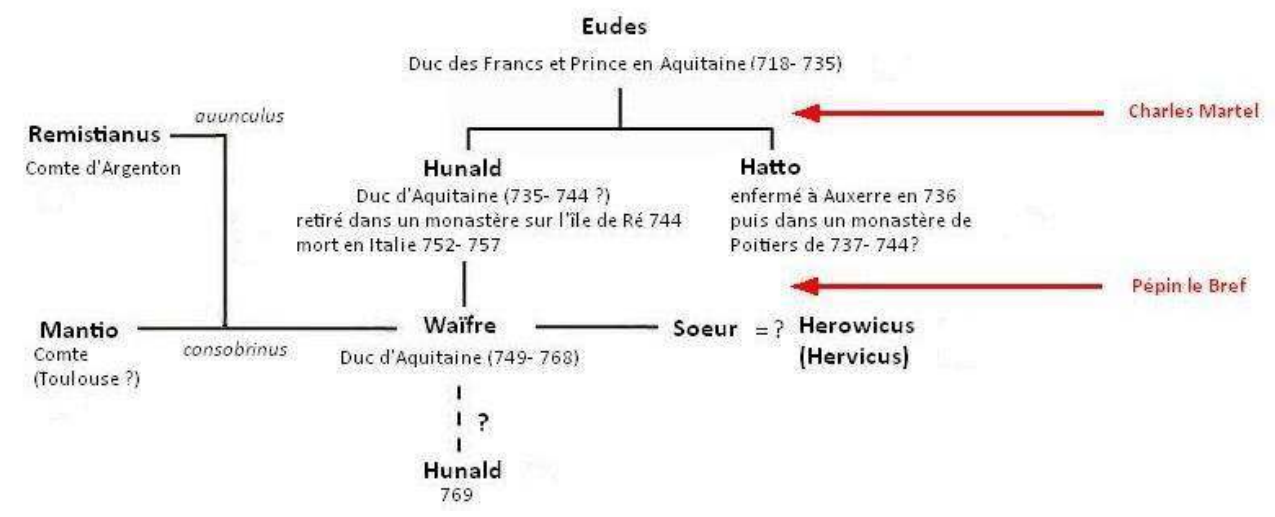

Figure - Les ducs d'Aquitaine et les interventions royales $\odot$ Adrien Bayard

\section{La construction d'un royaume}

7 L'évolution de ce discours doit être analysée en tenant compte des profonds changements survenus à la fin du VIIIe siècle. Les principautés périphériques sont alors réintégrées dans la dépendance directe des nouveaux rois des Francs et les dynasties ducales éliminées. Pour l'Aquitaine, cette mise au pas prend la forme d'un long conflit (760-769) entre la fin du règne de Pépin le Bref et les débuts de Charlemagne. La spécificité des espaces d'Outre-Loire dans ce processus provient de l'apparition d'un royaume d'Aquitaine pour le jeune Louis le Pieux dès $781^{26}$. C'est là un élément capital si l'on veut comprendre la structuration du discours des auteurs carolingiens sur l'Aquitaine, qui projettent sur les périodes antérieures une réalité de leur époque, l'existence d'une « nation » des Aquitani, dans un territoire défini et incarné par un roi.

8 L'un des éléments les plus tangibles, permettant de mesurer le contrôle royal des espaces, est la structuration d'un réseau palatial au sein duquel le souverain organise ses déplacements. Le palatium, symbole de l'exercice mobile du pouvoir, servait à la fois de résidence, de centre administratif, de lieu de production et de concentration des richesses et surtout de point de rencontre avec l'aristocratie. Une dizaine de sites palatiaux sont connus pour le royaume d'Aquitaine, tous situés entre Loire et Charente (figure 3$)^{27}$. La seconde caractéristique des palais aquitains provient de leur caractère urbain ou périurbain, formant un véritable binôme entre palais et citée ${ }^{28}$ tels Chasseneuil-Poitiers, Angeac-Angoulême, Le Pallet-Nantes ou bien directement implantés en ville comme à Bourges, Limoges et Poitiers. Les deux exceptions proviennent de Doué-la-Fontaine, véritable palais-frontière et d'Ebreuil, qui fait office de relais de chasse. Cette implantation au nord d'une Aquitaine s'étendant jusqu'à Barcelone s'explique par le contrôle des Carolingiens sur ces espaces, qui se manifeste par l'existence d'autres réseaux complétant, voire redoublant la trame palatiale. En effet, les souverains d'Aquitaine de Louis le Pieux à Carloman II (781-882) s'appuyèrent principalement sur le riche épiscopat de Poitiers, Limoges, Clermont, Bourges et de Bordeaux ${ }^{29}$. Les productions de leur chancellerie ${ }^{30}$ se concentrent également sur les pagi entre Loire et Garonne, où les rois encouragèrent les fondations monastiques et confirmèrent les possessions des grandes Églises franques ${ }^{31}$. Enfin, l'enracinement des grandes familles aristocratiques apparentées aux Carolingiens est particulièrement tangible dans ces régions. Ainsi les Garinides présents à Clermont depuis les années 820 laissent progressivement la place aux Guilhemides à partir de $870^{32}$. Cependant, ces réseaux ne sont absolument pas unifiés, 
ni également répartis dans l'espace. C'est justement la très grande hétérogénéité de ce royaume qui frappe le chercheur. On peut ainsi opposer le nord de l'Aquitaine, intégré au système carolingien, aux espaces compris entre Dordogne et Garonne. Dans ces zones bien moins documentées, les aristocrates semblent surtout fonder leur pouvoir sur le contrôle de sites fortifiés et sur de petites fondations monastiques. C'est auprès de ces élites non communicantes, pour reprendre l'expression de Martin Gravel ${ }^{33}$, que Pépin II trouve ses soutiens les plus fermes, car ces hommes avaient intérêt à maintenir un pouvoir royal proche et accessible. Enfin la Septimanie constitue un monde à part, où le pouvoir central intervient plus directement ${ }^{34}$. Or, l'une des raisons du succès des Guilhemides en Aquitaine pourrait justement provenir de leur capacité à évoluer entre ces différentes sphères ${ }^{35}$.

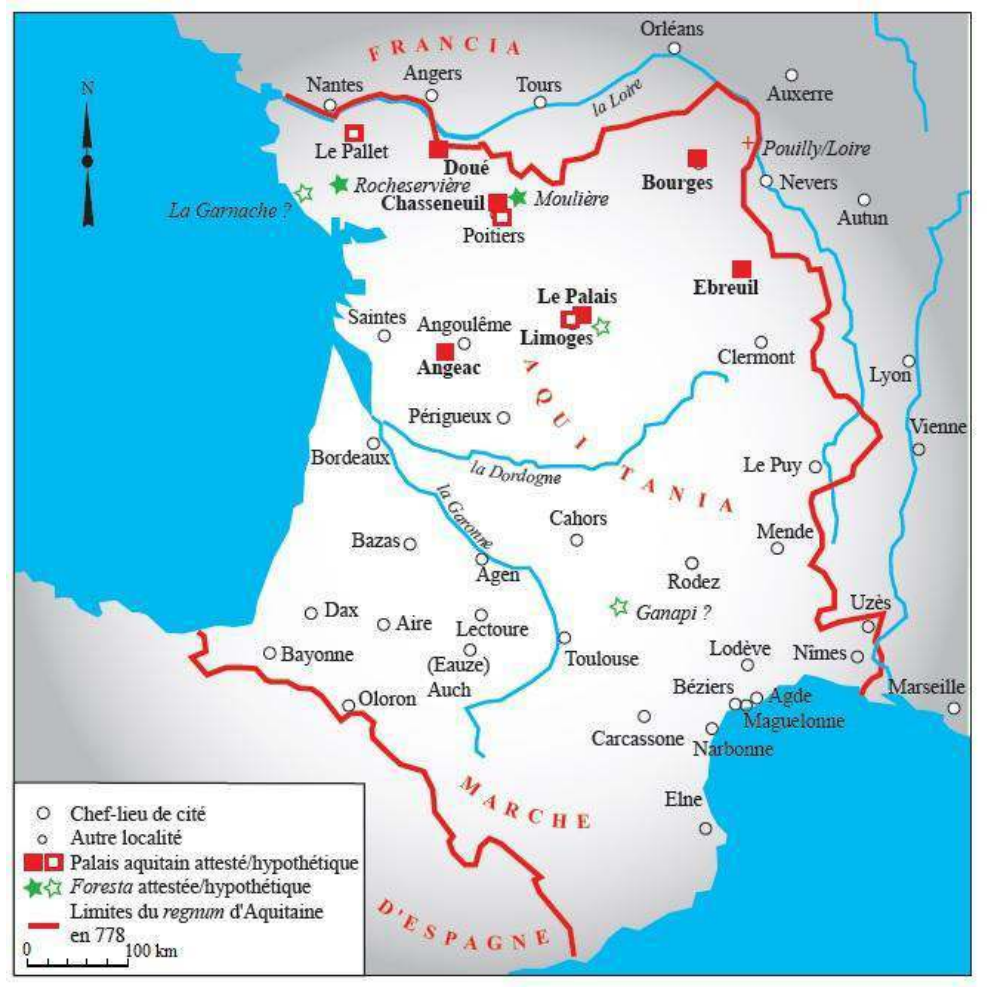

Figure - Le réseau des palais aquitains de Louis le Pieux à Charles le Chauve $\odot$ Luc Bourgeois

9 Ce bref tour d'horizon permet de mesurer la fragilité des processus d'élaboration d'une identité propre aux espaces entre Loire et Garonne durant le premier Moyen Âge. Ces derniers doivent être constamment replacés dans le cadre des évolutions à l'œuvre dans le reste de la Gaule, si l'on souhaite s'extraire d'un modèle téléologique. L'Aquitaine du haut Moyen Âge ne se résume pas à une province romaine cantonnée dans une résistance aux rois germaniques. On en vient alors à souligner le rôle joué par les pouvoirs centraux. Les souverains, loin de chercher à freiner ces transformations, participèrent à cet effort de définition d'une gens des Aquitains, en fonction des normes propres au monde franc. C'est par son inclusion directe dans le système carolingien que s'opère la transformation de la région, regio, au royaume, regnum, qui donne un semblant de corps à cette improbable nation des Aquitains. 


\section{NOTES}

1. Émile Mabille, Le Royaume d'Aquitaine et ses marches sous les carolingiens, Toulouse : Privat, 1870 ; Claude Perroud, Des origines du premier duché d'Aquitaine, Paris : Hachette, 1881; Léonce Auzias, L'Aquitaine carolingienne (778-987), Toulouse/Paris: Bibliothèque méridionale, 1937; Michel Rouche, L'Aquitaine des Wisigoths aux Arabes, 418-781, naissance d'une région, Paris: EHESS, 1979 ; Christian Lauranson-Rosaz, L'Auvergne et ses marges (Velay, Gévaudan), du VIII ${ }^{e}$ au XI ${ }^{e}$ siècle. La fin du monde antique?, Le Puy-en-Velay : Les cahiers de la Haute-Loire, 1987.

2. Isabelle Cartron, «L'Aquitaine 'après Vouillé' : réflexions sur la perception de l'espace dans les sources écrites franques", in : Luc Bourgeois (dir.), Wisigoths et Francs autour de la bataille de Vouillé (507), Recherches récentes sur le haut Moyen Âge dans le Centre-Ouest de la France, Actes des XXVIIIe Journées internationales d'archéologie mérovingienne Vouillé et Poitiers, Saint-Germain-en-Laye: AFAM, 2010, p. 41-54.

3. Grégoire de Tours, Decem libri historiarum, in : SS rer. Merov., Gregorii Turonensis opera, T. I, Bruno Krusch/Wilhelm Levison (éd.), Hannover: MGH, 1951, II, 25, p. 71 : Maxime tunc Novimpopulanae geminaeque Germaniae [Aquitaniae] urbes ab hac tempestate depopulatae sunt.

4. Sidoine Apollinaire, Carmina et Espistulae, André Loyen (éd. et trad.), Paris : Les Belles Lettres, 1970 (vol. 3), Ep., VII, 5, p. 42.

5. Grégoire de Tours, Decem libri historiarum, op.cit., V, 49, p. 262, 1.12-13: Cuius ingenium Turonicam urbem ab Arvernis populis emundavit.

6. Venance Fortunat, Opera poetica, in : Friedrich Leo (éd.), Auct. ant., T. 4, 1, Berlin: MGH, 1881, Praefatio, 4, p. 2, 1. 6 : Ligerem et Garonnam, Aquitaniae maxima fluenta transmittens.

7. Frédégaire, Chronicarum libri IV cum continuationibus, in: Bruno Krusch (éd.), SS rer. Merov., Hannover: MGH, 1888, I, 6, p. 23-24: Gentes autem quae linguas suas habent haec sunt: [...] Romani, qui et Latini, Lygires, Galli, qui et Celtae, Aquitani, Bruttanni, Hispani...

8. Ibid., c. 57, IV, p. 149 : Hoc tantum Chairiberto regendum concessit, quod et per pactiones vinculum estrinxit, ut amplius Airibertus nullo tempore adversus Dagobertum de regno patris repetire presumerit.

9. Ibid.

10. Claude Perroud, Des origines du premier duché d'Aquitaine, op. cit., p. 108-136.

11. Julien de Tolède, Historia rebellionis Pauli adversus Wambam, in: Bruno Krusch et Wilhelm Levison (éd.), SS rer. Merov., Passiones vitaeque sanctorum aevi merovingici, T.III, Hannover/Leipzig : MGH, 1910, p. 523.

12. Karl-Ferdinand Werner, «Les principautés périphériques dans le monde franc du VIIIe siècle ", in : I problemi dell'Occidente nel secolo VII, $\mathrm{t}^{\circ} 2$, Spoleto, 1973 : Settimane di studio del centro italiano di studi sull'alto Medioevo, XX, p. 483-514.

13. Edina Bozoky (dir.), Saints d'Aquitaine. Missionnaires et pèlerins du haut Moyen Âge, Rennes : PUR, 2010.

14. Passio Praejecti episcopi et matyris arverni, in: Bruno Krusch/Wilhelm Levison (éd.), SS rer. Merov., Passiones vitaeque sanctorum aevi merovingici, T.III, Hannover/Leipzig : MGH, 1910, p. 226, 1. 15-16: Igitur sanctus Preiectus Arvernensium provincia ortus est et Romane generis stemate praefulsit ; 1. 17-18 : qui originem duxere ex longinqua prosapia, catholicis viris, religionem christiane dignissimis, per quos etiam Dominus multa miracula declaravit.

15. Michel Rouche, "Peut-on parler d'une ethnogenèse des Aquitains? ", in ${ }^{\circ}$ : Herwig Wolfram/ Walter Pohl (dir.), Typen der Ethnogenese unter besonderer Berücksichtigung der Bayern I. Berichte des Symposions der Kommission für Frühmittelalterforschung, 27. bis 30. Oktober 1986, Stift Zwettl, 
Niederösterreich, Wien ${ }^{\circ}$ Veröffentlichungen der Kommission für Frühmittelalterforschung, t. 12, 1990, p. 45-51.

16. Alice Rio, Legal Practice and the Written Word in the Early Middle Ages. Frankish Formulae, c. 500-1000, Cambridge : Cambridge University Press, 2009.

17. Frédégaire, Chronicarum libri IV cum continuationibus, in : Olivier Devillers/Jean Meyers (trad.), Chroniques des temps mérovingiens, Livre IV et Continuations, Turnhout: Brepols, coll. Miroir du Moyen Âge, 2001, cont., 25, p. 228, 1. 4-9: Interea rebellantibus Wascones in regione Aquitania cum Chunoaldo duce, filio Eudone quondam, Carlomannus atque Pippinus germani principes, congregato exercito, Liger alveum Aurilianis urbem transeunt, Romanos proterunt, usque Beturgas urbem accedunt, suburbana ipsius igne conburent, Chunoaldo duce persequentes fugant, cuncta vastantes, Lucca castrum dirigunt atque funditus subvertunt, custodes illius castri capiunt; etenim victores existunt.

18. Karl-Ferdinand Werner, «Les principautés périphériques », art. cit.

19. Liber pontificalis, in : Michel Aubrun (trad.), Le Livre des papes (492-891), Turnhout : Brepols, coll. Miroir du Moyen Âge, 2007, Grégoire II, p. 104.

20. Annales Mettenses Priores, in: Bernhard von Simson (éd.), SS rer. Germ., T. 10, Hannover/ Leipzig : MGH, 1905, 735, p. 28.

21. Frédéric Le Roy/Saïd Yami (dir.), Stratégies de coopétition : rivaliser et coopérer simultanément, Bruxelles : De Boeck, 2010.

22. Miracula sancti Martialis Lemovicensis, in: Wilhelm Wattenbach (éd.), SS rer. Germ., T. 15, part. 1, Hannover : MGH, 1887, p. 280-281.

23. Cartulaire de Saint Julien de Brioude (Liber de honoribus sancto Juliano collatis), in : Henri Doniol (éd.), Mémoires de l'Académie des Sciences, Belles Lettres et Arts de Clermont-Ferrand, ClermontFerrand, 1861, T. 3, p. 53-463, ici n ${ }^{\circ} 25$, a. 757, p. 47-48.

24. Annales Mettenses Priores, op. cit., 744, p. 35.

25. Christian Lauranson-Rosaz, L'Auvergne et ses marges, op. cit., p. 42-43.

26. Annales regni Francorum inde ab a. 741 usque ad a. 829, qui dicuntur Annales Laurissenses maiores et Einhardi, in: Friedrich Kurze (éd.), SS rer. Germ., T. 6, Hannover: MGH, 1895, 751, p. 56-57; Philippe Wolff, "L'Aquitaine et ses marges », in: Helmut Beumann/Wolfgang Braunfels, (dir.), Karl der Große: Lebenswerk und Nachleben. Band I, Persönlichkeit und Geschichte, Düsseldorf: L. Schwann, 1965, p. 269-306.

27. Luc Bourgeois/Jean-François Boyer, «Les palais carolingiens d'Aquitaine: genèse, implantation et destin », in : Luc Bourgeois/Christian Remy (dir.), Demeurer, défendre et paraître: orientations récentes de l'archéologie des fortifications et des résidences aristocratiques médiévales entre Loire et Pyrénées, Chauvigny : APC (sous presse).

28. Josiane Barbier, «Les lieux de pouvoir en Gaule franque, l'exemple des palais », in : Caspar Ehlers, Places of Power - Orte der Herrschaf - Lieux du Pouvoir, Deutsche Königspfalzen, Göttingen : Vandenhoeck \& Ruprecht, 2007, p. 227-246.

29. Philippe Depreux, Prosopographie de l'entourage de Louis le Pieux (781-840), Sigmaringen: Thorbecke, 1997.

30. Recueil des actes de Pépin Irr et de Pépin II, rois d'Aquitaine (814-848), Léon Levillain (éd.)/Maurice Prou (dir.), Paris : Imprimerie Nationale, 1926.

31. Michel Rouche, L'Aquitaine, op. cit., p. 232 et 239-248.

32. Régine Le Jan, Famille et pouvoir dans le monde franc (VIIe-Xe siècles). Essai d'anthropologie sociale, Paris : Publications de la Sorbonne, 1995, p. 292 et 300.

33. Martin Gravel, Distances, rencontres, communications : réaliser l'Empire sous Charlemagne et Louis le Pieux, HAMA n 15, Turnhout : Brepols, 2012, p. 400-412.

34. Philippe Depreux, «Les préceptes pour les Hispani de Charlemagne, Louis le Pieux et Charles le Chauve », in : Philippe Sénac (dir.), Aquitaine-Espagne (VIIIe-XIIIe siècle), Poitiers : CESCM, 2001, p. 19-38. 
35. Adrien Bayard, Les Lieux élitaires dans l'Auvergne du premier Moyen Âge (thèse en cours), sous la direction de Régine Le Jan et de Joëlle Burnouf, Université Paris 1 Panthéon-Sorbonne.

\section{AUTEUR}

\section{ADRIEN BAYARD}

(Université Paris 1 Panthéon-Sorbonne et LAMOP (UMR 8589), ArScAn (UMR 7041)) 